\title{
Extend standardised methods and protocols for insect diet composition to insect
} energy and nutrient budgets

\author{
J. Casas ${ }^{1 *}$, T. Corbet $^{1}$, E. Desouhant ${ }^{2}$ and D. Giron ${ }^{1}$ \\ ${ }^{1}$ Institut de Recherche sur la Biologie de l'Insecte IRBI, UMR 7261, CNRS, Université de Tours, Tours, France; ${ }^{2}$ Université de \\ Lyon, Université Claude Bernard Lyon 1, CNRS, UMR 5558 Biométrie et Biologie Evolutive, Villeurbanne, 69622, France; \\ casas@univ-tours.fr
}

(c) 2020 Wageningen Academic Publishers

OPEN ACCESS CC) (1) (1) (-)

EDITORIAL

The community working on insects as feed and food recently moved towards a much more precise definition, use and reporting of insect diet composition. These efforts need to be matched by much more precise definition, use and reporting of its metabolic fate within the insect body, if the added workload is to fulfil its aims.

We regard the discussion paper by Bosch et al. (2020) in this journal as a key step in the domain of insects as food and feed (IFF). They propose a first set of standardised methods and protocols to quantify and report diet composition for the black soldier fly (BSF), meant to improve overall rigour and reproducibility. Indeed, without quantification, control and exact reporting of the diet composition, there is no hope of understanding and controlling nutrient and energy allocation in insects, for IFF or for any other purpose. Such understanding is also crucial for assessing the incidence of rearing procedures and related industrial processes on insect performance.

We argue that the efforts spelled out in Bosch et al. (2020) need to be extended beyond diet, into the analysis of the insect energy budget. Too many studies are still being published with the reporting of only one large class of nutrients, usually lipids in the case of the BSF, sometimes along with proteins. Insects are, however, biochemically highly plastic and can use several fuel types concurrently for a given task. Furthermore, interconnections between metabolic pathways abound and the hosted microbiota is notably expanding the biochemical palette. An energy budget uses chemical potential energy as a unit and typically equates the energy consumed with the sum of energy spent in reproduction, respiration, faeces and waste materials. So, the ultimate ideal nutrient and energy budget would consider all biochemical pathways of quantitative importance at any point in time.
A dynamic vision is indeed warranted, both for the diet and for the animal compositions as soon as the food composition varies and because both body composition and optimal energy requirements vary during insect ontogeny. Clearly, such a complete and dynamic nutrient and energy budget is too grandiose an aim, but any budget should eventually at least be balanced, i.e. input and outputs should be equal, once possible conversions have been considered. Many budgets are however not, to the point where some authors argue against the building of complete energy budgets (Davies and Hatcher, 1998): omission of terms (e.g. nutrient classes), too vague proxies (e.g. reporting nutrient ratios instead of amounts), and the uniqueness of the context in which they apply (e.g. having a single temperature regime) make them much less powerful than at first glance. We agree that many aspects and assumptions must be taken into account but disagree with the conclusion and instead encourage the community to quantify and report systematically at least the three major compartments, i.e. carbohydrates, lipids and proteins. If the interest is centred on lipids, glycogen should also be quantified as such, as energy storage can often be a dialogue between these two categories.

We briefly explain our rationale by starting with the concept of system determinacy. Underdetermination means that the number of equations is smaller than the number of unknowns. An infinite number of ways of combining the unknowns might lead to the same solution, or there may be no solution. In other words and in the IFF context, while we 
might know the identity of the variables of importance (i.e. the metabolites), the information at hand about the way they relate to each other (i.e. the equations) is not sufficient to hone in on a single solution. For example, if you want larvae to produce a given amount of lipids, knowing only the lipid amounts obtained directly from the diet is not sufficient. Amounts obtained through de novo lipogenesis from amino acids and carbohydrates must also be considered. Without a quantified relationship between them and the final amount of lipids expected in the insect's body, an infinite number of combinations is possible, precluding a systematic optimisation of the lipid production.

System determinacy is a plague to bio-modellers of metabolism, which do not even always recognise the importance of this very basic and key step (DiStefano III, 2015). This is not a theoretical digression, unrelated to insect energy metabolism. Indeed, the metabolic processes responsible for the largest energy consumption during embryogenesis of Drosophila are still unidentified (Song et al., 2019), for a developmental process otherwise well studied and for an organism exceedingly well studied. Another example is the recent discovery of urea being formed from uric acid through a functional amphibian-like uricolytic pathway, i.e. via allantoic acid, in mosquitoes. It was reported to be rather negligible in insects until recently (Scaraffia et al., 2008). It is now assumed to also be present in some parasitic wasps that are host-feeding, such as the well-studied Euplemus vuiletti (Richard et al., 2018). This finding implies that the original nitrogen budget of the wasp was incomplete, with some losses unaccounted for, i.e. it was also unbalanced (Casas et al., 2005, 2015). Given the use of proteins for many different tasks in this species, including maintenance, the entire nutrient budget must be revisited once the new metabolite (and related pathway) is included. This implies a new and lower estimation of lipid consumption for respiration, as proteins are also consumed for this purpose and in higher quantities than previously thought.

These two examples deal with insects outside those used so far for IFF but there is no reason to exclude the fact that similar findings may force us to rethink the nutritional ecology of IFF insect models besides well-characterised physiological pathways. The final example deals again with the BSF. The study of the role of carbohydrates is considered mandatory to understand BSF lipid metabolism in one of the most thorough studies published so far (Hoc et al., 2020). This finding, stemming from IFF, echoes the demonstration given by the many metabolic flux studies with other organisms: even the major and traditional metabolic pathways cannot be considered in isolation, but rather as part of a wider network. It is best to consider the network in its entirety to gain a full understanding of the metabolic state (Nikoloski et al., 2015). Often, additional conditions and relationships among unknowns can luckily reduce the dimensionality of the underdetermined problem, but cases in which a single solution is available do not really exist.

The community has recently matured towards a much more precise definition, use and reporting of diet composition. These efforts need to be matched by much more precise definition, use and reporting of its metabolic fate if the added work is to fulfil its aims. The inspiration for doing so can come from animal production with vertebrates on the one hand and metabolic engineering/systems biology with microorganisms and plants on the other. Systems biology and metabolic flux analysis have indeed gone a long way towards drawing meaningful and practical conclusions without having access to all metabolites of all pathways; these fields obviously have a lot to offer to the IFF community (see, for example, Stephanopoulos et al., 1998; Long and Antoniewicz, 2019; and the entire Volume 64 of Current Opinion in Biotechnology related to tracing metabolic flux: https://tinyurl.com/ yx9bwqj7). Considerations of life-history strategies and other evolutionary aspects may complement this dual approach. Indeed, many insects have complex life cycles and there are large, cross-over effects from one stage to the next in holometabolous insects (Llandres et al., 2015). Furthermore, tissue production seems to cost much less for holometabolous than hemimetabolous larvae (Ferral et al., 2020). Which species, which life stage and which stopping point in a production line are chosen for which purpose might therefore be based on such considerations.

IFF has no choice but to follow the research lines laid out in these different scientific fields, some out of them a long time ago. The alternative is a random walk in a highly multidimensional nutrient world, searching for elusive optimal locations. An integrative approach over biochemical pathways and life stages therefore seems preferable.

\section{Acknowledgements}

The Région Centre-Val de Loire MIMOSA project ( ${ }^{\circ} 2018$ 00124218) to DG funded the master thesis work done by TC, supervised by JC and ED, during which some of these ideas matured.

\section{References}

Bosch, G., Oonincx, D.G.A.B., Jordan, H.R., Zhang, J., Van Loon, J.J.A., van Huis, A. and Tomberlin, J.K., 2020. Standardisation of quantitative resource conversion studies with black soldier fly larvae. Journal of Insects as Food and Feed 6: 95-109. https://doi. org/10.3920/JIFF2019.0004

Casas, J., Body, M., Gutzwiller, F., Giron, D., Lazzari, C.R., Pincebourde, S., Richard, R. and Llandres, A. L., 2015. Increasing metabolic rate despite declining body weight in an adult parasitoid wasp. Journal of Insect Physiology 79: 27-35. 
Casas, J., Pincebourde, S., Mandon, N., Vannier, F., Poujol, R. and Giron, D., 2005. Lifetime nutrient dynamics reveal simultaneous capital and income breeding in a parasitoid. Ecology 86: 545-554.

Davies, M.S. and Hatcher, A.M., 1998. The energy budget: a useful tool? Annales Zoologici Fennici 35: 231-240.

DiStefano III, J., 2015. Dynamic systems biology modeling and simulation. Academic Press, Cambridge, MA, USA.

Ferral, N., Gomez, N., Holloway, K., Neeter, H., Fairfield, M., Pollman, K., Huang, Y.-W. and Hou, C., 2020. The extremely low energy cost of biosynthesis in holometabolous insect larvae. Journal of Insect Physiology 120: 103988.

Hoc, B., Genva, M., Fauconnier, M.L., Lognay, G., Francis, F. and Megido, R.C., 2020. About lipid metabolism in Hermetia illucens (L. 1758): on the origin of fatty acids in prepupae. Scientific Reports 10: 11916.

Llandres, A.L., Marques, G.M., Maino, J.L., Kooijman, S.A.L.M., Kearney, M.R. and Casas, J., 2015. A dynamic energy budget for the whole life-cycle of holometabolous insects. Ecological Monographs 85: 353-371.
Long, C.P. and Antoniewicz, M.R., 2019. High-resolution 13 C metabolic flux analysis. Nature Protocols 14: 2856-2877.

Nikoloski, Z., Perez-Storey, R. and Sweetlove, L.J., 2015. Inference and prediction of metabolic network fluxes. Plant Physiology 169: 1443-1455.

Richard, R., Foster, S., Giron, D. and Casas, J., 2018. A host-feeding wasp shares several features of nitrogen management with bloodfeeding mosquitoes. Journal of Insect Physiology 110: 1-5.

Scaraffia, P.Y., Tan, G., Isoe, J., Wysocki, V.H., Wells, M.A., Miesfeld, R.L., 2008. Discovery of an alternate metabolic pathway for urea synthesis in adult Aedes aegypti mosquitoes. Proceedings of the National Academy of Sciences 105: 518-523.

Song, Y., Park, J.O., Tanner, L., Nagano, Y., Rabinowitz, J.D. and Shvartsman, S.Y., 2019. Energy budget of Drosophila embryogenesis. Current Biology 29: R566-R567.

Stephanopoulos, G., Aristidou, A.A. and Nielsen, J., 1998. Metabolic engineering: principles and methodologies. Elsevier, Amsterdam, the Netherlands. 
